\title{
Nonlinear imaging condition and the effect of source illumination: Imaging fractures as nonwelded interfaces
}

\author{
Shohei Minato ${ }^{1}$ and Ranajit Ghose ${ }^{1}$
}

\begin{abstract}
Fluid flow through a fractured reservoir is often controlled by the large fractures. Seismically imaging these large fractures has the potential to illuminate their hydraulic properties. We derived a nonlinear imaging condition considering a medium containing nonwelded interfaces such as fractures for high-resolution fracture imaging. This was achieved by using the general correlation-type representation theorem relating the wavefield between two different states representing different fracture compliances. We numerically tested the imaging condition to investigate the effect of the nonlinear term and that of the one-sided source illumination. Assuming a dry fracture, we calculated the wavefield from a nonwelded interface. We obtained a P-wave imaging result from $\mathrm{P}$-wave sources. In the case of perfect source
\end{abstract}

\begin{abstract}
illumination, we found that introducing the nonlinear term in the imaging condition enhances the image because the nonwelded interface is imaged as a thin layer in an otherwise homogeneous medium. Investigating the effect of one-sided illumination by horizontally aligned sources revealed interesting limitations and possibilities. The imaging result for a horizontal fracture showed a volumetric distribution of nonzero amplitudes around the polarity change at the fracture that can be misinterpreted as a welded thick layer boundary. However, when the fracture was not horizontal, the imaging result was quite good and was closer to that with a perfect source illumination. These led to a new possibility of imaging subvertical fractures from surface seismic measurements, or subhorizontal fractures from vertical seismic profiling data, assuming that we successfully estimated the perturbed wavefield from the receiver responses.
\end{abstract}

\section{INTRODUCTION}

Seismic detection and characterization of large fractures are important because they dominate the hydraulic properties of a fractured reservoir (e.g., Aydin, 2000). Successful seismic applications are currently based on investigation of fracture-induced anisotropy using the effective medium theory with long-wavelength assumption (e.g., Bakulin et al., 2000). When the seismic wavelength is relatively short compared with the size of the target fractures, an incident wave generates scattered waves and the effective medium theory cannot be used. However, despite the wavelength being still too long to delineate the geometry of an individual fracture surface, the seismic scattering from a fracture can be approximated by a nonwelded interface boundary condition; e.g., a linear-slip boundary across which the stress is continuous but the displacement is discontinuous (e.g., Schoenberg, 1980; Pyrak-Nolte et al., 1990; Wapenaar et al., 2004). There are other models (e.g., Liu et al.,
2000) representing a single fracture; e.g., the thin-layer model or the model using microstructures (distribution of small cracks and contacts). We consider a nonwelded interface because this can represent the thin layers and microstructures in an average sense (e.g., Baik and Thompson, 1984; Hudson et al., 1997).

In this intermediate scale of seismic wavelength, Willis et al. (2006) and Fang et al. (2013) use scattered waves to obtain the spatial orientation and distribution of aligned multiple fractures. More recently, Minato and Ghose $(2013,2014)$ show that it is possible to obtain further details of the individual fractures from seismic scattering. They propose an approach to characterize the spatially heterogeneous elastic compliance along the fracture plane through wavefield extrapolation. The spatial heterogeneity along a fracture is the key determinant for fracture-associated hydraulic properties. This approach requires information on the position of fractures and hence a method to image the fractures with a high resolution.

\footnotetext{
Manuscript received by the Editor 25 August 2014; revised manuscript received 10 October 2014; published online 14 January 2015.

${ }^{1}$ Delft University of Technology, Delft, The Netherlands. E-mail: s.minato@tudelft.nl; r.ghose@tudelft.nl.

(C) 2015 Society of Exploration Geophysicists. All rights reserved.
} 
Fleury and Vasconcelos (2012) and Ravasi and Curtis (2013) propose a nonlinear imaging condition for high-resolution imaging of a boundary of contrasting acoustic or elastic constants. In this case, the imaging condition is a nonlinear function of the perturbed wavefield, in which the perturbed wavefield is defined as the difference between the total wavefield (in the true medium) and the background wavefield (in the reference medium). This new imaging condition allows achieving higher resolution in the migrated image than with the conventional imaging condition, which is linear with respect to the perturbed wavefield (Ravasi and Curtis, 2013). The nonlinear imaging condition is derived using the correlation-type reciprocity theorem applied to the perturbed wavefield. Then, the migration image can be seen as zero-offset and zero-time scattered amplitudes. Nonwelded interfaces such as fractures are not considered in these earlier studies. In this research, we have addressed this issue.

By including the effect of a nonwelded interface into the reciprocity theorem appropriately, a general correlation-type representation theorem can be obtained (Wapenaar, 2007). Based on this, we have derived a new, nonlinear imaging condition for a medium containing nonwelded interfaces. The imaging condition is an integral equation with the sources located along a closed surface encompassing the fracture. However, exploration seismic applications are often limited by partial or one-sided illumination, in which the sources are located only on the surface of the earth. Investigating the effect of partial source illumination on the result of fracture imaging using the nonlinear imaging condition is crucial for evaluating the feasibility of this approach.

In this paper, we first derive the nonlinear imaging condition from the general correlation-type representation theorem. Next, we investigate numerically the importance of the nonlinear term in the derived imaging condition, in the case of perfect source illumination. We discuss the difference in the imaging result as compared with conventional thick-layer imaging. Finally, we look into the effect of one-sided source illumination on imaging a fracture with different dips, using the newly derived nonlinear imaging condition.

Here, we focus on a single nonwelded interface in a homogeneous medium. The field data, however, may contain the effect of multiple fractures of different scales. The proposed method can image fractures with a size comparable with or larger than the seismic wavelength. Therefore, the size limit of the imagable fracture will depend on the frequency; e.g., large faults from low-frequency measurements in deep seismology, joints from high-frequency mea-
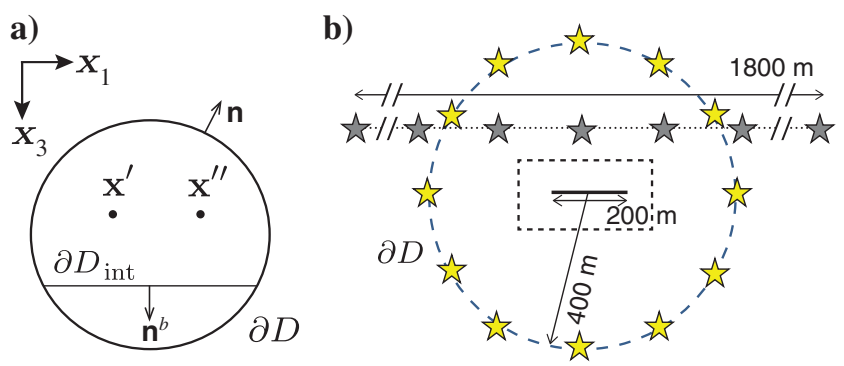

Figure 1. (a) Configuration for the general correlation-type representation theorem. The $\partial D_{\text {int }}$ indicates a nonwelded interface, $\mathbf{n}$ and $\mathbf{n}^{b}$ are vectors normal to $\partial D$ and $\partial D_{\text {int }}$, respectively and (b) fracture and source distribution on a vertical plane in the numerical experiments, for perfect source illumination (yellow stars) and for onesided illumination (gray stars). surements in shallow seismic exploration, or small fractures in laboratory experiments. A cluster of unimagably small fractures may be treated as a homogeneous, effectively anisotropic medium (e.g., Bakulin et al., 2000).

\section{THEORY}

The boundary condition for a nonwelded interface can be written in the general form as (Wapenaar et al., 2004)

$$
[\mathbf{M u}]=-j \omega \mathbf{Y}\langle\mathbf{M u}\rangle,
$$

where $[\cdot]$ and $\langle\cdot\rangle$ indicate the jump and the average, respectively, across the nonwelded interface. For the elastic wave, we have $\mathbf{u}^{T}=$ $\left(\mathbf{v}^{T},-\boldsymbol{\tau}_{1}^{T},-\boldsymbol{\tau}_{2}^{T},-\boldsymbol{\tau}_{3}^{T}\right)$, where $\mathbf{v}$ and $\boldsymbol{\tau}_{i}$ are the particle velocity and the traction vectors. The matrices in equation 1 are defined as

$$
\mathbf{M}=\left(\begin{array}{cccc}
\mathbf{I} & \mathbf{0} & \mathbf{0} & \mathbf{0} \\
\mathbf{0} & n_{1}^{b} \mathbf{I} & n_{2}^{b} \mathbf{I} & n_{3}^{b} \mathbf{I}
\end{array}\right) \quad \text { and } \quad \mathbf{Y}=\left(\begin{array}{cc}
\mathbf{0} & \mathbf{S}^{b} \\
\boldsymbol{\rho}^{b} & \mathbf{0}
\end{array}\right)
$$

where the superscript $b$ denotes the boundary parameters; that is, $n_{i}^{b}, \mathbf{S}^{b}$, and $\boldsymbol{\rho}^{b}$ are the vector normal to the interface, the boundary compliance tensor, and the boundary density tensor, respectively. The linear-slip boundary condition (Schoenberg, 1980) is a special case of a nonwelded interface with the boundary parameters $\left(\mathbf{n}^{b}\right)^{T}=(0,0,1)^{T}, \mathbf{S}^{b}=\operatorname{diag}\left(\eta_{T}, \eta_{T}, \eta_{N}\right)$, and $\boldsymbol{\rho}^{b}=\mathbf{0}$, where $\eta_{T}$ and $\eta_{N}$ are the tangential and the normal fracture compliance, respectively. Here, we assume a horizontal fracture and a rotationally invariant compliance matrix (Schoenberg, 1980).

The nonlinear imaging condition is derived from the correlationtype representation theorem that relates the wavefield between two different states. We use the general representation theorem (equation 59 in Wapenaar, 2007) that includes the effect of a nonwelded interface and consider two positions $\mathbf{x}^{\prime}$ and $\mathbf{x}^{\prime \prime}$ inside a closed surface $\partial D$ (Figure 1a). In the case of a horizontal fracture with a rotationally invariant compliance matrix, the general correlation-type representation theorem in frequency domain yields to

$$
\begin{aligned}
& \bar{v}_{i, j}^{*}\left(\mathbf{x}^{\prime}, \mathbf{x}^{\prime \prime}\right)+v_{i, j}\left(\mathbf{x}^{\prime}, \mathbf{x}^{\prime \prime}\right)= \\
& -\oint_{\partial D}\left[\bar{v}_{m, i}^{*}\left(\mathbf{x}, \mathbf{x}^{\prime}\right) \tau_{m n, j}\left(\mathbf{x}, \mathbf{x}^{\prime \prime}\right)+v_{m, j}\left(\mathbf{x}, \mathbf{x}^{\prime \prime}\right) \bar{\tau}_{m n, i}^{*}\left(\mathbf{x}, \mathbf{x}^{\prime}\right)\right] n_{n} \mathrm{~d}^{2} \mathbf{x} \\
& +j \omega \int_{\partial D_{\text {int }}}\left[\Delta \eta_{T} \bar{\tau}_{13, i}^{*}\left(\mathbf{x}, \mathbf{x}^{\prime}\right) \tau_{13, j}\left(\mathbf{x}, \mathbf{x}^{\prime \prime}\right)+\Delta \eta_{T} \bar{\tau}_{23, i}^{*}\left(\mathbf{x}, \mathbf{x}^{\prime}\right) \tau_{23, j}\left(\mathbf{x}, \mathbf{x}^{\prime \prime}\right)\right. \\
& \left.+\Delta \eta_{N} \bar{\tau}_{33, i}^{*}\left(\mathbf{x}, \mathbf{x}^{\prime}\right) \tau_{33, j}\left(\mathbf{x}, \mathbf{x}^{\prime \prime}\right)\right] \mathrm{d}^{2} \mathbf{x}
\end{aligned}
$$

where we assume that the two states have identical elastic constants but different fracture compliances. Here, $\bar{v}_{i, j}\left(\mathbf{x}^{\prime}, \mathbf{x}^{\prime \prime}\right)$ and $v_{i, j}\left(\mathbf{x}^{\prime}, \mathbf{x}^{\prime \prime}\right)$ indicate $i$ th component of particle velocity at $\mathbf{x}^{\prime}$ due to $j$ th direction of point force at $\mathbf{x}^{\prime \prime}$ in two different states, respectively; $\bar{\tau}_{p q, i}$ and $\tau_{p q, j}$ are the corresponding stress tensors, and $\Delta \eta_{T}$ and $\Delta \eta_{N}$ are perturbation in tangential and normal compliances between two different states. We assume the compliances to be real-valued functions. A real-valued fracture compliance implies that the total elastic energy is conserved during wave scattering; i.e., anelastic attenuation is ignored. Fracture models involving elastic thin layers or distribution of cracks result in real-valued fracture compliances (e.g., Baik and Thompson, 1984; Hudson et al., 1997). However, the presence 
of a viscous fluid or the occurrence of plastic behavior at the asperities on a fracture surface may cause the fracture compliance to be complex valued (e.g., Pyrak-Nolte et al., 1990; Yoshioka and Kikuchi, 1993). The second integral in equation 3 is taken along (any number of) nonwelded interfaces defined as $\partial D_{\text {int }}$. Note that as we have assumed identical elastic constants between the two states, equation 3 does not contain any contrast of elastic constants.

As in Vasconcelos et al. (2009), we assume the total wavefield to be $v_{i, j}=v_{i, j}^{0}+v_{i, j}^{S}$, where $v_{i, j}^{0}$ and $v_{i, j}^{S}$ are the background wavefield and the perturbed wavefield due to the nonzero compliance perturbation, respectively. When we consider the two states in equation 3 to be both perturbed fields, $\Delta \eta_{T}$ and $\Delta \eta_{N}$ vanish, and we have

$$
\begin{aligned}
& v_{i, j}^{S *}\left(\mathbf{x}^{\prime}, \mathbf{x}^{\prime \prime}\right)+v_{i, j}^{S}\left(\mathbf{x}^{\prime}, \mathbf{x}^{\prime \prime}\right)= \\
& -\oint_{\partial D}\left[v_{m, i}^{0 *}\left(\mathbf{x}, \mathbf{x}^{\prime}\right) \tau_{m n, j}^{S}\left(\mathbf{x}, \mathbf{x}^{\prime \prime}\right)+v_{m, j}^{0}\left(\mathbf{x}, \mathbf{x}^{\prime \prime}\right) \tau_{m n, i}^{S *}\left(\mathbf{x}, \mathbf{x}^{\prime}\right)\right] n_{n} \mathrm{~d}^{2} \mathbf{x} \\
& -\oint_{\partial D}\left[v_{m, i}^{S *}\left(\mathbf{x}, \mathbf{x}^{\prime}\right) \tau_{m n, j}^{0}\left(\mathbf{x}, \mathbf{x}^{\prime \prime}\right)+v_{m, j}^{S}\left(\mathbf{x}, \mathbf{x}^{\prime \prime}\right) \tau_{m n, i}^{0 *}\left(\mathbf{x}, \mathbf{x}^{\prime}\right)\right] n_{n} \mathrm{~d}^{2} \mathbf{x} \\
& -\oint_{\partial D}\left[v_{m, i}^{S *}\left(\mathbf{x}, \mathbf{x}^{\prime}\right) \tau_{m n, j}^{S}\left(\mathbf{x}, \mathbf{x}^{\prime \prime}\right)+v_{m, j}^{S}\left(\mathbf{x}, \mathbf{x}^{\prime \prime}\right) \tau_{m n, i}^{S *}\left(\mathbf{x}, \mathbf{x}^{\prime}\right)\right] n_{n} \mathrm{~d}^{2} \mathbf{x} .
\end{aligned}
$$

This equation is the basis for the nonlinear imaging condition that we propose here. It indicates that the perturbed wavefield at any position inside $\partial D$ can be retrieved from the values along the closed surface $\partial D$. Equation 4 is the same as that in Ravasi and Curtis (2013); however, these authors do not consider a nonwelded interface. We obtain the same equation due to our assumption of the compliances to be real-valued functions. Although we have considered horizontal fractures to derive equation 3, equation 4 is valid for fractures of any arbitrary orientation and dip because $\Delta \eta_{T}$ and $\Delta \eta_{N}$ always vanish. Note that for complex-valued fracture compliances, $\Delta \eta_{T}$ and $\Delta \eta_{N}$ do not vanish and the imaginary part of the compliances remains in the integral along $\partial D_{\text {int }}$.

By collapsing $\mathbf{x}^{\prime}$ and $\mathbf{x}^{\prime \prime}$ at the same point (image point $\mathbf{x}_{P}$ ) and by summing over frequencies, an imaging condition that represents an estimate of the zero-time and zero-offset amplitude of the perturbed wavefield can be derived. To explicitly see the effect of P- and Swaves, we can replace the particle velocity $v_{i, j}^{0, S}$ by the potential field $G_{\Psi_{0}, \Psi_{0}}^{0, S}$ (Wapenaar and Fokkema, 2006; Halliday et al., 2012), with the source and receiver represented by the P-wave potential $\Psi_{0}$. Furthermore, when a $\mathrm{P}$-wave source is activated and no $\mathrm{S}$-wave source is available as in the case of most marine seismic surveys, ignoring the terms containing the $S$-wave potential in equation 4 gives an approximate imaging condition for P-wave due to only $\mathrm{P}$-wave sources:

$$
\begin{aligned}
& I_{\mathrm{PP}}\left(\mathbf{x}_{\mathrm{P}}\right)=\int_{-\infty}^{\infty} G_{\Psi_{0}, \Psi_{0}}^{S}\left(\mathbf{x}_{\mathrm{P}}, \mathbf{x}_{\mathrm{P}}\right) \mathrm{d} \omega \\
& \approx \frac{2}{\rho c_{\mathrm{P}}} \int_{0}^{\infty} \oint_{\partial D}\left[G_{\Psi_{0}, \Psi_{0}}^{0 *}\left(\mathbf{x}_{\mathrm{P}}, \mathbf{x}\right) G_{\Psi_{0}, \Psi_{0}}^{S}\left(\mathbf{x}_{\mathrm{P}}, \mathbf{x}\right)\right] \mathrm{d}^{2} \mathbf{x} \mathrm{d} \omega \\
& +\frac{2}{\rho c_{\mathrm{P}}} \int_{0}^{\infty} \oint_{\partial D}\left[G_{\Psi_{0}, \Psi_{0}}^{S *}\left(\mathbf{x}_{\mathrm{P}}, \mathbf{x}\right) G_{\Psi_{0}, \Psi_{0}}^{0}\left(\mathbf{x}_{\mathrm{P}}, \mathbf{x}\right)\right] \mathrm{d}^{2} \mathbf{x} \mathrm{d} \omega \\
& +\frac{2}{\rho c_{\mathrm{P}}} \int_{0}^{\infty} \oint_{\partial D}\left[G_{\Psi_{0}, \Psi_{0}}^{S *}\left(\mathbf{x}_{\mathrm{P}}, \mathbf{x}\right) G_{\Psi_{0}, \Psi_{0}}^{S}\left(\mathbf{x}_{\mathrm{P}}, \mathbf{x}\right)\right] \mathrm{d}^{2} \mathbf{x} \mathrm{d} \omega,
\end{aligned}
$$

where $c_{P}$ indicates the $\mathrm{P}$-wave velocity on the boundary $\partial D$. Note that equation 5 is derived already after far-field approximation.

One can see that the first and the second integrals in equation 5 contain the crosscorrelation between the background wavefield and the perturbed wavefield, which indicates that they are linear with respect to the perturbed wavefield. However, the third integral contains the autocorrelation of the perturbed wavefield, which means that it is nonlinear with respect to the perturbed wavefield. In real applications, the background wavefield $G_{\Psi_{0}, \Psi_{0}}^{0}$ and the perturbed wavefield $G_{\Psi_{0}, \Psi_{0}}^{S}$ on the right side of equation 5 may be estimated by calculating the wavefield using a macrovelocity model and by extrapolating the receiver responses, respectively.

\section{NUMERICAL MODELING}

We test the derived nonlinear imaging condition for fractures, using 2D numerical modeling. In this study, we particularly focus on the source illumination and on the contribution of the nonlinear term in the imaging condition to resolution. Therefore, we assume that the background and perturbed wavefields are already estimated, and we ignore the discussion on extrapolation of receiver responses.

\section{Imaging result in the case of perfect source illumination}

Here, we will show that the introduction of the nonlinear term leads to canceling undesirable sidelobes in the result of the conventional linear imaging condition and enhances the image. In this subsection, we consider perfect illumination of a nonwelded interface by seismic sources: 40 transient point $\mathrm{P}$-wave sources being located on a closed surface $\partial D$ of radius $400 \mathrm{~m}$ (yellow stars in Figure 1b). A 200 -m-long horizontal fracture is located inside $\partial D$ (Figure 1b). The values of are taken from earlier field experiments (Worthington and Hudson, 2000). We assume a dry fracture; i.e., $\eta_{N}=\eta_{T}=1.1 \times$ $10^{-9} \mathrm{~m} / \mathrm{Pa}$. The homogeneous background represents sandstone with $V_{\mathrm{P}}=4000 \mathrm{~m} / \mathrm{s}, V_{\mathrm{S}}=2350 \mathrm{~m} / \mathrm{s}$, and $\rho=2500 \mathrm{~kg} / \mathrm{m}^{3}$. We calculate the seismic response using a $100 \mathrm{~Hz}$ Ricker wavelet, and the procedure of Coates and Schoenberg (1995) is modified to use a rotated staggeredgrid finite-difference time-domain method (Saenger et al., 2000), which implicitly incorporates the linear-slip boundary condition.

Figure 2 shows the result with the conventional linear imaging condition (the first and the second integrals in equation 5), with the nonlinear term only (the third integrals in equation 5), and with the nonlinear imaging condition (summation of Figure $2 a$ and $2 b$ ), respectively, using all sources (yellow stars in Figure 1b). The imaging results for an area $200 \mathrm{~m}$ in height and $400 \mathrm{~m}$ in width encompassing the fracture are shown in Figure 2 (for the dashed area in Figure 1b). The result with the conventional linear imaging condition (Figure 2a) shows that the peak negative amplitudes (cold color) are correctly located along the fracture position. However, the artifact due to the correlation of the incident wave with the transmitted wave remains above and below the fracture. The nonlinear term (Figure $2 b$ ) has opposite polarity (warm color) to that of the artifact, and hence stacking these two results in a higher resolution (Figure 2c). The amplitude in the imaging result should be zero everywhere at a wavelength or more distance from the fracture because the nonlinear imaging condition corresponds to a zero-time amplitude at zero-offset data, as shown in the previous section. In Figure 2c, we indeed notice that the nonwelded interface is imaged as a single wavelet in an otherwise almost-zero-amplitude area. The 
a)

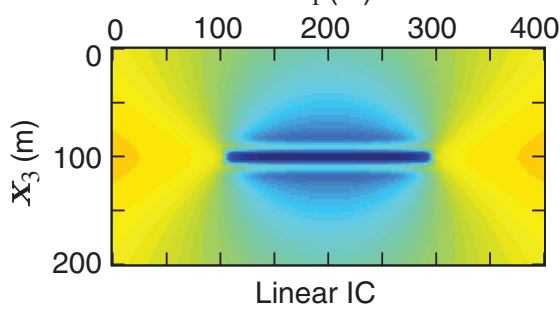

c)

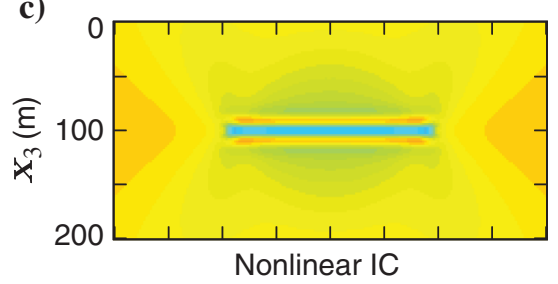

b)

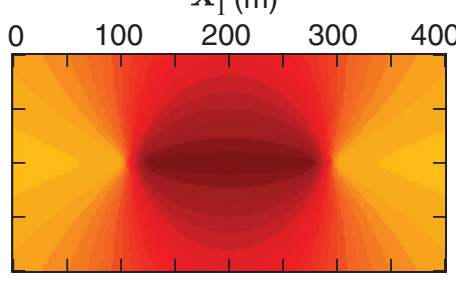

Nonlinear term

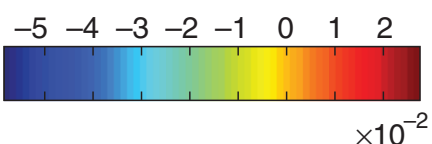

Figure 2. Results of imaging using various imaging conditions, in the case of perfect source illumination. (a) Conventional linear imaging condition, (b) using only the nonlinear term, and (c) nonlinear imaging condition.

Figure 3. Imaging results in the case of (b-f) onesided illumination (seismic sources only at the surface) of a fracture with different dips. For comparison, in (a) the imaging result for a horizontal fracture in the case of a perfect source illumination is shown. In all cases here, the nonlinear imaging condition is applied. (g) The imaged amplitude plotted along a line perpendicular to the fracture (dashed line in [a-f]) shows more clearly the differences. a)

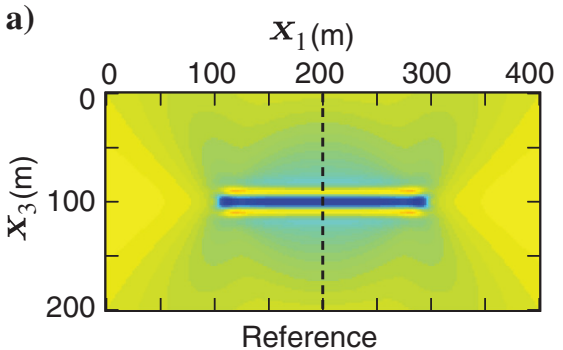

c)

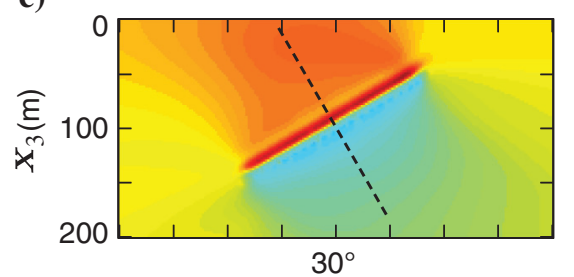

e)

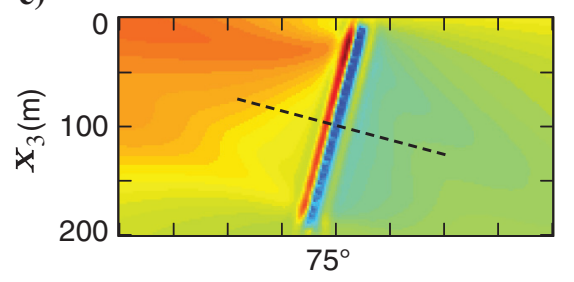

amplitude around the fracture is not exactly zero, possibly because we ignore the S-wave sources.

The imaging result for a nonwelded interface using the nonlinear imaging condition (Figure 2c) is different from that of a welded boundary of a thick layer, where the nonzero amplitude is volumetrically distributed in the perturbed zone (see, e.g., Figure 21 in Ravasi and Curtis, 2013). This is because when one uses the homogeneous background velocity, the scattered (perturbed) wavefield from the welded thick layer is originated from a volumetric distribution of the medium perturbation (or surface distribution in the case of a $2 \mathrm{D}$ configuration). Because the imaging result is the zero-time amplitude at zero-offset data, the result for the welded thick layer shows a volumetric distribution of the nonzero amplitude in the perturbed zone. In contrast, the scattered wavefield from the nonwelded interface is originated from a surface distribution (or line distribution in the case of a $2 \mathrm{D}$ configuration) of the medium pertur-

g)

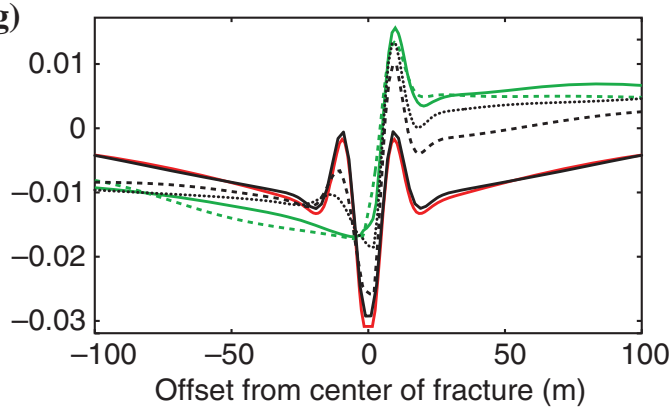

Reference - f)

d)
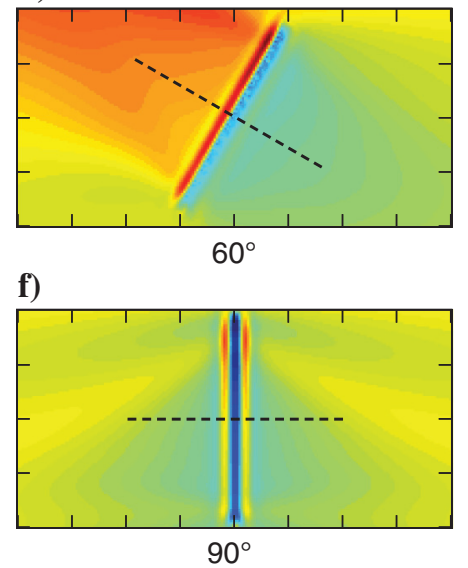

$60^{\circ}$
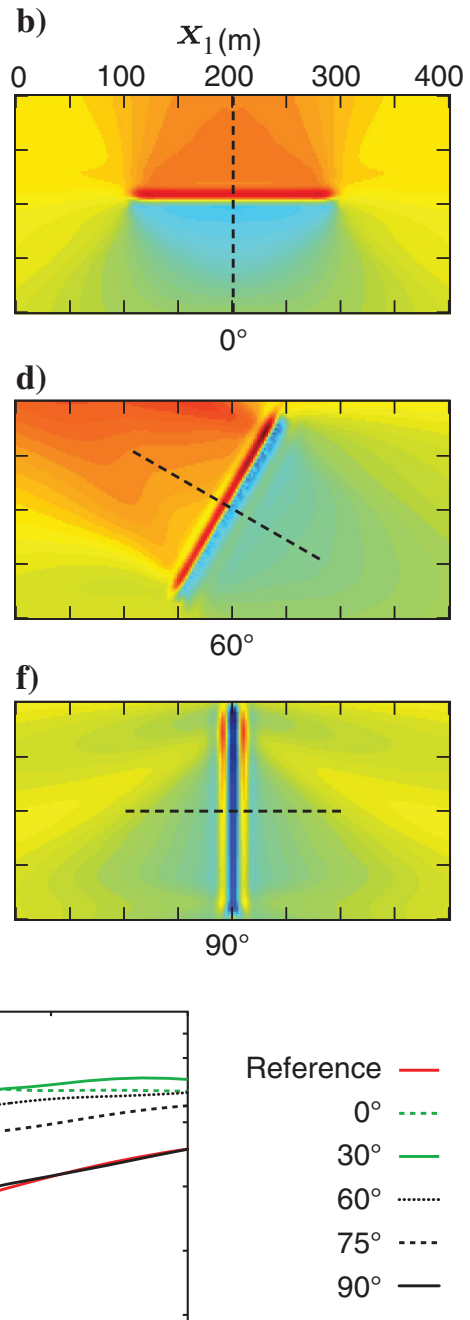
bation; the imaging result shows a surface distribution of the nonzero amplitude. However, due to frequency-band limitation of the source wavelet, the imaging result of the nonwelded interface exhibits the interface as a thin but volumetric distribution of nonzero amplitudes (Figure 2c). This imaging result may be similar to that of a welded thin layer in an otherwise homogeneous medium. However, the key difference of a nonwelded interface from a welded thin layer is that, for a nonwelded interface, one cannot split an apparent thin layer into several thinner layers, even if the data have a much broader frequency band.

\section{Effect of one-sided source illumination}

Perfect source illumination is rarely possible in exploration geophysical measurements. Therefore, we investigate the effect of one-sided illumination by installing sources along a horizontal line located $200 \mathrm{~m}$ above the fracture (gray stars in Figure 1b), simulating a surface seismic experiment. Along a 1800-m-long profile, 46 sources are installed at $40 \mathrm{~m}$ intervals. Within the dashed box in Figure 1b, we position a 200-m long fracture and we experiment with different fracture dips.

Figure 3 shows the result of the nonlinear imaging condition with different fracture dips $\left(0,30,60,75\right.$, and $\left.90^{\circ}\right)$. For comparison, the result in the case of perfect source illumination is shown in Figure $3 \mathrm{a}$, for a horizontal fracture. The amplitudes in Figure $3 \mathrm{a}-3 \mathrm{f}$ are normalized by the root-mean-square amplitudes. Because we estimate $\mathrm{PP}$ image ( $\mathrm{P}$ source and $\mathrm{P}$ receiver), Figure $3 \mathrm{~b}-3 \mathrm{f}$ is expected to show the same imaging result as in Figure 3a, except for the effect of fracture dip, in case the source illumination were perfect. However, Figure 3b-3f shows marked differences compared with Figure 3a, indicating the effect of one-sided source illumination.

In the imaging result for the horizontal fracture (Figure 3b), the polarity change perpendicular to the fracture is clear: a positive or warm-colored area above the fracture and a negative or cool-colored area below the fracture. Furthermore, the nonzero amplitudes are more volumetrically distributed than those in the case of perfect illumination (Figure 3a). Therefore, this can be misinterpreted as a thick-layer boundary, as discussed in the previous subsection. The polarity change at the fracture implies that the source illumination from below the fracture may cancel the volumetric distribution of the nonzero amplitudes. When the fracture is vertical (Figure 3f), the result is close to that in the case of a perfect illumination (Figure 3a). This is because for a one-sided illumination from horizontally aligned sources, vertical fractures are illuminated from both sides, which produces imaging results that are close to that with a perfect illumination. Note that as the dip of the fracture changes from horizontal to vertical, the amplitude difference between the two sides of the fracture decreases and the shape of the imaged wavelet approaches to that with a perfect source illumination. This can be seen more clearly if the amplitude along a line perpendicular to the fracture (at the center of the fracture) is examined (Figure $3 \mathrm{~g}$ ).

\section{CONCLUSION}

We derive a nonlinear imaging condition for high-resolution imaging of large fractures and to investigate the effect of one-sided source illumination. The fractures are generalized as nonwelded interfaces and represented by the linear-slip boundary condition. Unlike previous studies, which do not consider fracture as nonwelded interfaces, we show that also for nonwelded interfaces, the imaging condition can be retrieved from a general correlation-type representation theorem by assuming the fracture compliances to be real valued. For numerical tests, we calculate the wavefield from a nonwelded interface assuming a dry fracture. We first test the nonlinear imaging condition when the seismic sources surround the fracture completely. The results show that the nonlinear term in the imaging condition contributes to canceling the artifacts that appear in the conventional linear-imaging condition, and the fracture is imaged as a thin layer in an otherwise homogeneous medium.

Investigating next the effect of one-sided illumination (horizontally aligned surface sources) on imaging a dipping fracture reveals that the imaging result of a vertical or near-vertical fracture using the nonlinear imaging condition is closer to the result for perfect source illumination. This finding is important because it suggests the possibility of imaging subvertical fractures from surface seismic measurements or subhorizontal fractures from vertical seismic profiling data, assuming that we have successfully estimated the perturbed wavefield. In an actual field data application, the estimation of the perturbed wavefield will require extrapolation of the receiver responses. The effect of receiver configuration on the estimation accuracy remains to be evaluated in the future.

\section{ACKNOWLEDGMENTS}

This work is supported by The Netherlands Research Centre for Integrated Solid Earth Science.

\section{REFERENCES}

Aydin, A., 2000, Fractures, faults, and hydrocarbon entrapment, migration and flow: Marine and Petroleum Geology, 17, 797-814, doi: 10.1016/ S0264-8172(00)00020-9.

Baik, J.-M., and R. B. Thompson, 1984, Ultrasonic scattering from imperfect interfaces: A quasi-static model: Journal of Nondestructive Evaluation, 4, 177-196, doi: 10.1007/BF00566223.

Bakulin, A., V. Grechka, and I. Tsvankin, 2000, Estimation of fracture parameters from reflection seismic data. Part I: HTI model due to a single fracture set: Geophysics, 65, 1788-1802, doi: 10.1190/1.1444863.

Coates, R., and M. Schoenberg, 1995, Finite difference modeling of faults and fractures: Geophysics, 60, 1514-1526, doi: 10.1190/1.1443884.

Fang, X., M. C. Fehler, Z. Zhu, Y. Zheng, and D. R. Burns, 2013, Reservoir fracture characterization from seismic scattered waves: Geophysical Journal International, 196, 481-492, doi: 10.1093/gji/ggt381.

Fleury, C., and I. Vasconcelos, 2012, Imaging condition for nonlinear scattering-based imaging: Estimate of power loss in scattering: Geophysics, 77, no. 1, S1-S18, doi: 10.1190/geo2011-0135.1.

Halliday, D., A. Curtis, and K. Wapenaar, 2012, Generalized $P P+P S=S S$ from seismic interferometry: Geophysical Journal International, 189, 1015-1024, doi: 10.1111/j.1365-246X.2012.05396.x.

Hudson, J. A. E. Liu, and S. Crampin, 1997. The mean transmission properties of a fault with imperfect facial contact: Geophysical Journal International, 129, 720-726, doi: 10.1111/j.1365-246X.1997.tb04507.x.

Liu, E., J. Hudson, and T. Pointer, 2000, Equivalent medium representation of fractured rock: Journal of Geophysical Research, 105, 2981-3000, doi: 10.1029/1999JB900306.

Minato, S., and R. Ghose, 2013, Inverse scattering solution for the spatially heterogeneous compliance of a single fracture: Geophysical Journal International, 195, 1878-1891, doi: 10.1093/gji/ggt348.

Minato, S., and R. Ghose, 2014, Imaging and characterization of a subhorizontal non-welded interface from point source elastic scattering response: Geophysical Journal International, 197, 1090-1095, doi: 10.1093/gji/ ggu037.

Pyrak-Nolte, L., L. Myer, and N. Cook, 1990, Transmission of seismic waves across single natural fractures: Journal of Geophysical Research, 95, 8617-8638, doi: 10.1029/JB095iB06p08617.

Ravasi, M., and A. Curtis, 2013, Nonlinear scattering based imaging in elastic media: Theory, theorems, and imaging conditions: Geophysics, 78, no. 3, S137-S155, doi: 10.1190/geo2012-0286.1.

Saenger, E. H., N. Gold, and S. A. Shapiro, 2000, Modeling the propagation of elastic waves using a modified finite-difference grid: Wave Motion, 31 77-92, doi: 10.1016/S0165-2125(99)00023-2. 
Schoenberg, M., 1980, Elastic wave behavior across linear slip interfaces: Journal of the Acoustical Society of America, 68, 1516-1521, doi: 10 $.1121 / 1.385077$.

Vasconcelos, I., R. Snieder, and H. Douma, 2009, Representation theorems and Green's function retrieval for scattering in acoustic media: Physical Review E, 80, 036605, doi: 10.1103/PhysRevE.80.036605.

Wapenaar, K., 2007, General representations for wavefield modeling and inversion in geophysics: Geophysics, 72, no. 5, SM5-SM17, doi: 10 $.1190 / 1.2750646$.

Wapenaar, K., and J. Fokkema, 2006, Green's function representations for seismic interferometry: Geophysics, 71, no. 4, SI33-SI46, doi: 10.1190/1 .2213955 .
Wapenaar, K., E. Slob, and J. Fokkema, 2004, Reciprocity and power balance for piece-wise continuous media with imperfect interfaces: Journal of Geophysical Research, 109, B10301, doi: 10.1029/2004JB003002.

Willis, M., D. Burns, R. Rao, B. Minsley, M. Toksoz, and L. Vetri, 2006, Spatial orientation and distribution of reservoir fractures from scattered seismic energy: Geophysics, 71, no. 5, O43-O51, doi: 10.1190/1.2235977.

Worthington, M. H., and J. A. Hudson, 2000, Fault properties from seismic Q: Geophysical Journal International, 143, 937-944, doi: 10.1046/j.1365246X.2000.00315.X.

Yoshioka, N., and M. Kikuchi, 1993, Visco-elastic response of joints to transmission waves: Geophysical Research Letters, 20, 1143-1146, doi: 10.1029/93GL01260. 